from the tip of the new growth straight through the substrate on which the material is growing.

Tracking the surface evolution of the material provides insight into how the structure evolves and helps researchers understand the nanostructure formation mechanism. The creation of surface pole figures was particularly important in understanding the growth of nanoblades, as the surface morphology changed greatly with time.

The surface pole figure technique was first outlined by Tang, a postdoctoral research associate in Wang's group, in a 2006 issue of Applied Physics Letters. In that article, surface pole figures were created for nanorod growth. The researchers are now working to analyze nanoblade growth to provide additional insight into the growth patterns of these nanomaterials.

\section{Researchers Look at Nanotubes Inside Living Animals}

T.K. Leeuw, K.M. Beckingham, R.B. Weisman, and colleagues at Rice University have confirmed that nearinfrared fluorescent imaging was capable of detecting DNA-sized, single-walled carbon nanotubes inside living fruit flies.

"Carbon nanotubes are much smaller than living cells, and they give off fluorescent light in a way that researchers hope to harness to detect diseases earlier than currently possible," said Bruce Weisman, professor of chemistry at Rice. "In order to do that, we need to learn how to detect and monitor nanotubes inside living tissues, and we must also determine whether they pose any hazards to organisms."

Researchers have studied how carbon nanotubes interact with tissues of rabbits, mice, and other animals, but Weisman and co-researcher Kathleen Beckingham, professor of biochemistry and cell biology, chose the fruit fly Drosophila melanogaster to attempt the detection of nanotubes inside a living animal.

In the study, published in the September issue of Nano Letters (p. 2650; DOI: $10.1021 /$ nl0710452), fruit fly larvae were raised on a yeast paste that contained carbon nanotubes. The flies were fed this food from the time they hatched throughout their initial feeding phase of 4-5 days. Fruit flies are ravenous eaters during this period and gain weight continuously until they are about 200 times heavier than hatchlings. Then they become pupae. As pupae, they do not eat or grow. They mature inside pupal cases and emerge as adult flies.

"Developmentally, the first few days of a fruit fly's life are critical," Beckingham said. "We provided larval flies with a steady diet of food that contained carbon nanotubes and checked their weight just after they emerged from their pupal cases. We found no significant differences in the adult weight of nanotube-fed flies when compared to control groups that were not fed carbon nanotubes."

The nanotube-fed larvae also survived to adulthood just as well as the control group.

Using a custom-built microscope, the team aimed a red laser beam into the fruit flies. This excited a fluorescent glow from the carbon nanotubes as they emitted nearinfrared light of specific wavelengths. The researchers were then able to use a camera to view the glowing nanotubes inside living flies. Videos constructed from these images showed peristaltic movements in the digestive system.

When the researchers removed and examined tissues from the flies, they found the near-infrared microscope enabled them to see and identify individual nanotubes inside the tissue specimens. The highest concentration of nanotubes was found in the dorsal vessel, which is analogous to a main blood vessel in a mammal. Lesser concentrations were found in the brain, ventral nerve cord, salivary glands, trachea, and fat. Based on their assays, the team estimates that only about 1 in 100 million nanotubes passed through the gut wall and became incorporated into the flies' organs.

The researchers said that the "apparent biocompatibility of single-walled carbon nanotubes shown here supports their promise for the development of novel biomedical applications."

\section{Researchers Probe a "Quantum Paramagnetic" Spin State}

A team of international researchers that includes researchers has found experimental evidence of a highly sought-after type of arrangement of atomic magnetic moments, or spins, in a series of materials. Their work, one of few studies of this particular spin state that has been postulated as a possible underlying mechanism for high-temperature superconductivity, may eventually serve as a test of current and future theoretical models of exotic spin states.

At the National Institute of Standards and Technology (NIST) Center for Neutron Research (NCNR) and the HahnMeitner Institute in Berlin, Germany, the researchers used intense beams of neutrons to probe a series of antiferromagnets, materials in which each spin cancels another, giving the material a net magnetic field of zero. The results, described in the August 26 posting of Nature Materials
(DOI:10.1038/nmat1986), revealed evidence of a rare and poorly understood "quantum paramagnetic" spin state, in which neighboring spins pair up to form "entangled spin singlets" that have an ordered pattern and enable the material to weakly respond to an outside magnetic field (i.e., become paramagnetic).

The antiferromagnets used in this work are composed mainly of zinc and copper and are distinguished by their proportions of each, with the number of copper ions determined by the number of zinc ions. At the atomic level, the material is formed of many repeating layers. The atoms of each layer are arranged into a structure known as a kagome lattice, which is a pattern of triangles laid point-to-point whose basic unit resembles a six-point star.

Researchers have been studying antiferromagnets with kagome structures for the last 20 years because they suspected these materials harbored interesting spin structures. But good model systems, like the zinc/copper compounds used by this group, had not been identified.

At the NCNR, the researchers determined how varying concentrations of zinc and copper and varying temperatures affected fluctuations in the way the spins are arranged in these materials. Using a neutron spectrometer at the Hahn-Meitner Institute, they also investigated the effect of external magnetic fields of varying strengths. The group uncovered several magnetic phases in addition to the quantum paramagnetic state and were able to construct a complete phase diagram as a function of the zinc concentration and temperature. They are planning further experimental and theoretical studies to learn more about the kagome system.

This work was led by S.-H. Lee at the University of Virginia. The other participating institutions are the University of Fukui in Japan and the Technical University of Berlin.

\section{Use of Electric Field and Nanoscale Nozzle Achieves Significant Resolution in E-Jet Printing}

By combining electrically induced fluid flow with nanoscale nozzles, researchers J.-U. Park and J.A. Rogers of the University of Illinois, Urbana-Champaign (UIUC), S.J. Kang of the Korea Research Institute of Standards and Science, and their colleagues at the UIUC Center for Nanoscale Chemical Electrical Mechanical Manufacturing Systems have established new benchmarks for precision control and resolution in jet-printing processes.

"We have invented methods for an electrohydrodynamic jet (e-jet) printing 